\title{
Generation of clinical-grade human induced pluripotent stem cells in Xeno-free conditions
}

Juan Wang ${ }^{1,2+}$, Jie Hao ${ }^{1 \dagger}$, Donghui Bai ${ }^{1}$, Qi Gu', Weifang Han ${ }^{1,3}$, Lei Wang ${ }^{1}$, Yuanqing Tan ${ }^{1,3}$, Xia Li $i^{1,2}$, Ke Xue ,

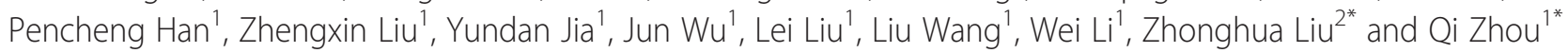

\begin{abstract}
Introduction: Human induced pluripotent stem cells (hiPSCs) are considered as one of the most promising seed cell sources in regenerative medicine. Now hiPSC-based clinical trials are underway. To ensure clinical safety, cells used in clinical trials or therapies should be generated under GMP conditions, and with Xeno-free culture media to avoid possible side effects like immune rejection that induced by the Xeno reagents. However, up to now there are no reports for hiPSC lines developed completely under GMP conditions using Xeno-free reagents.

Methods: Clinical-grade human foreskin fibroblast (HFF) cells used as feeder cells and parental cells of the clinical-grade hiPSCs were isolated from human foreskin tissues and cultured in Xeno-free media. Clinical-grade hiPSCs were derived by integration-free Sendai virus-based reprogramming kit in Xeno-free pluriton ${ }^{\mathrm{TM}}$ reprogramming medium or X medium. Neural cells and cardiomyocytes differentiation were conducted following a series of spatial and temporal specific signals induction according to the corresponding lineage development signals. Biological safety evaluation of the clinical-grade HFF cells and hiPSCs were conducted following the guidance of the "Pharmacopoeia of the People's Republic of China, Edition 2010, Volume III".
\end{abstract}

Results: We have successfully derived several integration-free clinical-grade hiPSC lines under GMP-controlled conditions and with Xeno-free reagents culture media in line with the current guidance of international and national evaluation criteria. As for the source of hiPSCs and feeder cells, biological safety evaluation of the HFF cells have been strictly reviewed by the National Institutes for Food and Drug Control (NIFDC). The hiPSC lines are pluripotent and have passed the safety evaluation. Moreover, one of the randomly selected hiPSC lines was capable of differentiating into functional neural cells and cardiomyocytes in Xeno-free culture media.

Conclusion: The clinical-grade hiPSC lines therefore could be valuable sources for future hiPSC-based clinical trials or therapies and for drug screening.

\section{Introduction}

Human pluripotent stem cells (hPSCs) can differentiate into any type of cells in the body, such as functional neural progenitor cells or cardiomyocytes, and therefore have enormous value in regenerative medicine. The increasing incidence of degenerative diseases, limitations of traditional

\footnotetext{
* Correspondence: liu086@163.com; zhouqi@ioz.ac.cn

${ }^{\dagger}$ Equal contributors

${ }^{2}$ College of Life Science, Northeast Agricultural University of China, Harbin 150030, China

'State Key of Stem Cells and Laboratory of Reproductive Biology, Institute of Zoology, Chinese Academy of Sciences, Beijing 100101, China

Full list of author information is available at the end of the article
}

therapeutic methods, and the shortage of isolated human functional cells have urged scientists to turn to stem cellbased cell replacement therapies. Although the translation from basic discoveries to clinical settings comes with great challenges, intensive stem cell-based clinical trials are emerging from around the world. For human embryonic stem cells ( $\mathrm{hESCs}$ ), a clinical trial of spinal-cord injury treatment using immature glial cells derived from hESCs by the Geron Corporation (Menlo Park, California, USA) has recommenced after it was brought to a halt in 2011 [1]. Another clinical trial of hESCs involving the generation of retinal pigmented epithelial (RPE) cells for the treatment of eye disorders such as Stargardt's macular dystrophy, myopic 
macular degeneration, and advanced dry age-related macular degeneration is currently being conducted by the Advanced Cell Technology company (Marlborough, Massachusetts, USA) in America [2]. The mid-term outcomes confirmed the safety and efficacy of hESC-derived RPE in patients [3]. When taking moral and ethical aspects into consideration, human induced pluripotent stem cells (hiPSCs) are more ideal and feasible cell sources for transplantation compared with $\mathrm{hESCs}$. A clinical trial for eye disorder treatment using hiPSC-derived RPE cells is also now being carried out in Japan [4].

Initially, the generation of hiPSCs involved integrated retrovirus expressing Oct4, Sox2, Klf4, and $c-M y c[5,6]$. However, random integrations may result in insertional mutagenesis consequently risking patients' safety. Also, unexpected activation of the integrated oncogene $c-M y c$ may initiate tumorigenesis [7]. To circumvent the aforementioned problems, integration-free hiPSCs have been generated using Sendai viruses [8], episomal vectors [9], mRNAs [10], minicircle DNAs [11], microRNAs [12], and proteins [13]. Although each method has its own merits and disadvantages, integration-free reprogramming methods are optimal for future clinical applications.

Most of the hESC lines collected by the National Institutes of Health (NIH) have been reported ineligible for future therapeutic products use because their derivation processes did not follow the "Tissue Donor Guidance" [14]. Precautionary actions are therefore of utmost importance in order to ensure the safety, effectiveness, traceability, reproducibility, and legality of hiPSCs intended for clinical trials or therapies. Careful screening for legal and eligible donors is a very important step. According to the current national and international regulation policies, most countries require a good manufacturing practice (GMP) environment when handling the cells $[15,16]$. Reagents used in the culture process will greatly affect the safety and quality of the cells. Xeno reagents would not only increase the risk of infections but also cause immune rejection upon cell transplantation [17]. Almost all countries have advocated that animal reagents should not be used in cells for clinical applications [18]. Therefore it is sensible to use Xeno-free reagents in all cell handling processes. To further ensure the safety of the cells used in clinical settings, endotoxin and serious pathogenic microorganism such as mycoplasma and HIV virus have to be tested [19].

We define hiPSCs intended to be used for potential clinical applications as clinical-grade hiPSCs. Theoretically, clinical-grade hiPSCs should meet the following requirements. First, parental cell donors should meet the requirements of the "Tissue Donor Guidance". Second, the cell handling processes should be conducted under GMP-controlled environments and with Xeno-free reagents. Third, the derived clinical-grade hiPSCs should be integration-free and biologically safe.
Previously, a group reported the derivation of hiPSCs under fully defined conditions. However, the biological safety of the obtained hiPSC lines is questionable. Furthermore, the group did not describe whether their protocol was within the "Tissue Donor Guidance" [20]. Several other groups have also successfully achieved the so-called clinical-grade hiPSC lines by converting the existing nonclinical-grade hiPSC lines. Still, in these processes animal products were applied in the initial stages, which may confer risks of cross-species infectious agent transfer [21-23]. For these reasons, there is an urgent need to derive clinical-grade hiPSC lines strictly following all requirements of clinical-grade hiPSCs.

Here, we report successful generation of several clinicalgrade hiPSC lines. A series of pathogenic microorganism tests were conducted on foreskin tissue donors according to the "Tissue Donor Guidance" of China. The human foreskin fibroblast (HFF) cells used for reprogramming and as feeder cells were isolated and cultured using Xenofree reagents in a GMP-grade laboratory and were confirmed negative for mycoplasma and certain pathogenic microorganisms. Furthermore, the biological safety of the HFF cells is validated by the National Institutes for Food and Drug Control (NIFDC). All processes of the hiPSC generation were conducted under GMP-controlled conditions using Xeno-free media and reagents. The resulting hiPSCs are validated as integration-free, and are able to randomly differentiate into three germ layers in vitro and in vivo as well as directly differentiate into functional neural cells and cardiomyocytes in Xeno-free media. The biological safety of these hiPSCs is further validated. These hiPSC lines, generated following all of the requirements of clinical-grade hiPSCs strictly, might serve as valuable cell sources for future clinical applications.

\section{Methods}

\section{Ethical approval}

The foreskin tissues were donated by males undergoing foreskin operation in Beijing Children's Hospital with the approval of the Animal and Medical Ethics Committee of Institute of Zoology, Chinese Academy of Sciences. Written consent was obtained from the donors' parents. The content of the consent includes the potential use of cells derived from the foreskin (research or potential clinical therapies) and the rights and obligations of the donors. After donation, a thorough assessment of the donors' medical history and infectious diseases were performed. Based on the results, donors were selected. All information is secured to protect the privacy and confidentiality of donors. No financial benefits were involved in the donation process. All cell isolation and culture procedures followed closely the guidelines legislated and posted by the Ministry of Health of the People's Republic of China. 
All animal experiments were approved in advance by the Animal and Medical Ethics Committee of Institute of Zoology, Chinese Academy of Sciences.

\section{Isolation of fibroblasts from foreskin tissues}

Foreskin tissues were collected into $50 \mathrm{ml}$ centrifuge tubes containing Dulbecco's Phosphate Buffered Saline (DPBS)CTS (Life Technologies, Carlsbad, California, USA) and transported to the laboratory on ice. After sufficient washing with DPBS-CTS, fat and connective tissues were removed from the foreskin. The foreskin was then cut into small pieces with a volume of about $1 \mathrm{~mm}^{3}$ and transferred to T75 flasks precoated with MesenCult-XF attachment substrates (Stem Cell Technologies, Vancouver, British Columbia, Canada) without cell culture medium. After 24 hours of inverted culturing in a humidified incubator (at $37{ }^{\circ} \mathrm{C}, 5 \% \mathrm{CO}_{2}$ ), $4 \mathrm{ml}$ FibroGRO ${ }^{\mathrm{m}}$ Xeno-Free Human Fibroblast Expansion Medium (Millipore, Billerica, Massachusetts, USA) was added to each flask. After 7 days of culture, fibroblasts could outgrow the tissues and be routinely passaged. The obtained fibroblasts were used as parental cells for hiPSC induction and as feeder cells after being irradiated with gamma rays.

\section{Cell culture}

HFF cells were cultured in FibroGRO ${ }^{\text {mat }}$ Xeno-Free Human Fibroblast Expansion Medium (Millipore) on dishes precoated with Mesencult-XF attachment substrates according to the manufacturer's instructions. Clinical-grade hiPSCs were initially cultured on gamma ray-inactivated human feeder cells in pluriton ${ }^{\mathrm{Tm}}$ reprogramming medium (Stemgent, Lexington, Massachusetts, USA) or X medium (configured in our laboratory). The formulations of $\mathrm{X}$ medium were as follows: clinical-grade hESC basic medium and clinical-grade N2B27 medium mixed at 1:1 ratio supplemented with $10 \mathrm{ng} / \mathrm{ml}$ human leukemia inhibitory factor (hLif; Peprotech) and human basic fibroblast growth factor (bFGF; Peprotech, Rocky Hill, Rocky Hill, USA). The clinical-grade hESC basic medium contained knockout Dulbecco's modified Eagle's medium (DMEM)-CTS (Life Technologies), $20 \%$ knockout serum replacement (KOSR)-CTS (Life Technologies), $100 \mu \mathrm{M}$ none essential amino acid (NEAA; Life Technologies), $2 \mathrm{mM}$ GlutaMAX-CTS (Life Technologies), $55 \mu \mathrm{M}$ $\beta$-mercaptoethanol (Life Technologies), and $1000 \mathrm{U} / \mathrm{ml}$ penicillin/streptomycin (Life Technologies). The clinicalgrade N2B27 medium was a mixture of knockout DMEMCTS supplemented with N2-CTS (Life Technologies) and Neurobasal Medium-CTS (Life Technologies) supplemented with B27-CTS (Life Technologies) at 1:1 ratio plus $100 \mu \mathrm{M}$ NEAA, $2 \mathrm{mM}$ GlutaMAX-CTS, $55 \mu \mathrm{M} \beta-$ mercaptoethanol, and $1000 \mathrm{U} / \mathrm{ml}$ penicillin/streptomycin. At passages 10-12, the cells were transferred to E8 medium (Life Technologies) without feeder cells.

\section{Induction of hiPSCs from HFF cells}

HFF cells were cultured in six-well sterile culture plates precoated with MesenCult-XF Attachment Substrate in

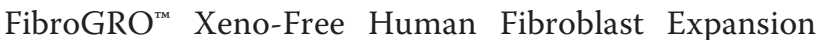
Medium without antibodies. The CytoTune ${ }^{\circ}$-iPS Sendai Reprogramming Kit (Life Technologies) was used for deriving integration-free iPSCs according to the manufacturer's instructions. The medium used in the reprogramming process was pluriton ${ }^{\text {tx }}$ reprogramming medium or $\mathrm{X}$ medium. Colonies with typical hPSC morphology were picked 20 days post transduction for further expansion.

\section{Alkaline phosphatase staining}

Alkaline phosphatase staining was performed using the Alkaline Phosphatase Assay Kit (Beyotime, Shanghai, China) according to the manufacturer's instructions.

\section{Immunofluorescence staining}

Immunofluorescence staining was conducted as described previously [23]. The primary antibodies used were OCT4 (1:200; Santa, Dallas, Texas, USA), SOX2 (1:200; Millipore), SSEA-1 (1:200; Millipore), SSEA-3 (1:200; Millipore), SSEA-4 (1:200; Millipore), TH1 (1:400; Millipore), TUJ1 (1:1000; Covance, Princeton, New Jersey, USA), NKX2.5 (1:200; Abcam, Cambridge, UK), and CTNT (1:200; R\&D, Minneapolis, Minnesota, USA). The secondary antibodies were donkey anti-mouse IgG $(\mathrm{H}+\mathrm{L})$ conjugated to Cy3 (1:200; Jackson, Lansing, Michigan, USA), donkey anti-mouse IgG conjugated to Cy5 (1:200; Jackson), donkey anti-goat IgG conjugated to Cy5 (1:200; Jackson), donkey anti-rabbit IgG conjugated to fluorescein isothiocyanate (FITC) (1:200; Jackson), and donkey anti-rat IgG conjugated to Cy5 (1:200; Jackson).

\section{Karyotyping and G-binding}

When hiPSCs reached 60-70\% confluency, karytoyping and G-binding analysis were carried out by Chinese Academy of Medical Science \& Peking Union Medical College.

\section{RNA isolation, reverse transcription and PCR analysis}

RNA isolation and reverse transcription were performed as described previously [24]. Real-time PCRs were conducted with the SYBR ${ }^{\circ}$ Premix EX Taq ${ }^{\mathrm{Ti}}$ kit (Takara, Minamikusatsu Station, Japan) and Agilent, Santa Clara, California, USA, Mx3005P equipment according to the manufacturer's instructions. Normal PCR reactions were conducted according to the previous report [24]. Primer sequences used in the experiments are presented in Additional file 1.

\section{Embryoid body formation assay}

The embryoid body (EB) formation assay was conducted as described previously [24] with the exception of using Xeno-free ethylenediamine tetraacetic acid (EDTA; Life Technologies) for cell dissociation and clinical-grade hESC basic medium for EB culture. 


\section{Teratoma formation assay}

Confluent cells in a six-well plate were harvested using EDTA and suspended in DPBS-CTS. Then $10^{6}$ cells in 20-30 $\mu \mathrm{l} \mathrm{DPBS}$ were injected into each testis of severe combined immunodeficiency (SCID) mouse under a sterile stereo microscope. The mice were sacrificed 8 weeks after injection for teratoma examination following the guidelines of the Institutional Animal Care and Use Committee (IACUC). The teratomas were fixed, sliced, and stained with hematoxylin and eosin for further analysis.

\section{Neural cell differentiation}

Neural cell differentiation was performed as described previously [25] apart from the media used being Xeno-free in all processes. Briefly, confluent hiPSCs were dissociated into single cells by tryple-CTS (Life Technologies) and plated on Vitronectin-coated dishes at a density of $4 \times 10^{5}$ cells $/ \mathrm{cm}^{2}$ on day 0 . Initially, the cells were cultured in a medium of knockout DMEM-CTS supplemented with $15 \%$ KOSR-CTS, $100 \mu$ M NEAA, 2 mM GlutaMAX-CTS, $55 \mu \mathrm{M} \beta$-mercaptoethanol, and $1000 \mathrm{U} / \mathrm{ml}$ penicillin/streptomycin. On day 5 , the medium containing KOSR-CTS was gradually transitioned to N2 medium-CTS as described previously [25]. On day 11 , the medium was changed to Neurobasal medium-CTS supplemented with $2 \%$ B27-CTS. To induce differentiation, growth factors and small molecules were added as follows: $10 \mu \mathrm{M}$ SB431542 (Stemgent) from day 0 to 5, $100 \mathrm{nM}$ LDN93189 (Stemgent) from day 0 to 11, $3 \mu \mathrm{M}$ CHIR99021 from day 3 to $13,100 \mathrm{ng} / \mathrm{ml} \mathrm{SHH} \mathrm{C25II}$ (R\&D), $2 \mu \mathrm{M}$ purmorphamine (Stemgent), and $100 \mathrm{ng} / \mathrm{ml}$ FGF8 (R\&D) from day 1 to 7 , and $20 \mathrm{ng} / \mathrm{ml}$ brain-derived neurotrophic factor (BDNF; R\&D), $0.2 \mathrm{mM}$ ascorbic acid (Sigma, Louis, Missouri, USA), $20 \mathrm{ng} / \mathrm{ml}$ glial cell linederived neurotrophic factor (GDNF; R\&D), $1 \mathrm{ng} / \mathrm{ml}$ transforming growth factor type $\beta 3$ (TGFß3; R\&D), $0.5 \mathrm{mM}$ dibutyryl cAMP (Sigma), and $10 \mu \mathrm{M}$ (DAPT) N-[(3,5Difluorophenyl)acetyl]-L-alanyl-2-phenyl]glycine-1,1-dimethylethyl ester (Tocris, Bristol, Bristol, UK) from day 11 to 23.

\section{Cardiomyocyte differentiation}

Confluent hiPSCs were digested into single cells by accutase (Life Technologies) and seeded on vitronectincoated dishes at a density of $1.25 \times 10^{5}$ cells $/ \mathrm{cm}^{2}$. Then the cells were grown in E8 medium for 2-3 days to reach $100 \%$ confluency. To improve the efficiency of single cell survival, $10 \mu \mathrm{M}$ Y27632 were added on the first day. Culture medium used in the differentiation process was RPMI 1640 (Life Technologies) with a combination of chemically defined components (modified in our laboratory and data not published) which we named Car-CDM. From day 0 (the day for differentiation medium adding) to day 8 , Car-CDM without insulin was used as differentiation medium. In this period, the medium was changed on days $0,1,3$, and 5 . From day 0 to $1,4 \mu \mathrm{M}$ were added, whereas $2 \mu \mathrm{M}$ Wnt-C59 (Sigma) was added from day 3 to 8 (Sigma). From day 8 onward, the differentiation medium was changed to Car-CDM plus insulin and the medium was changed every other day up to day 14 . Beating cardiomycytes generally appeared on day 12 of differentiation.

\section{Endotoxin testing}

Endotoxin testing was performed using the ToxinSensor ${ }^{\mathrm{mm}}$ Gel Clot Endotoxin Assay Kit (GeneScript, Piscataway, New Jersey, USA) according to the manufacturer's instructions.

\section{Mycoplasma testing}

Mycoplasma testing was performed using the PlasmoTest ${ }^{\mathrm{Tu}}$ Reagent Kit (InvivoGen, San Diego, California, USA) according to the manufacturer's instructions.

\section{Pathogenic microorganism testing}

The defined pathogenic microorganisms tested were human papilloma virus (HPV), human parvovirus B19, human immunodeficiency virus (HIV), John Cunningham virus (JCV), Epstein-Barr virus (EBV), human hepatitis $\mathrm{C}$ virus (HCV), human hepatitis A virus (HAV), human cytomegalovirus (HCMV), human T-lymphotropic virus I (HTLV-I), human hepatitis B virus (HBV), bovine virus, and porcine virus. These viruses were detected using corresponding kits produced by Zhongshandaan Company, Guangzhou, Guangdong, China. Some of the viruses were reviewed by Beijing Ai Puyi Medical Testing Center, Beijing, China. The hiPSCs were also inoculated into the chorioallantoic membrane and yolk sac of chicks according to the methods described in the Chinese Pharmacopoeia (third edition).

\section{Results}

Preparations for generation of clinical-grade hiPSCs

Safety, traceability, and reproducibility are three basic criteria for cells to be used in clinical trials or therapies. To meet these criteria, GMP environments are required for the whole process of cell manipulation. We have established a GMPcontrolled cell culture laboratory and it has passed the Beijing Institute for Drug Control (Beijing Center for Health Food and Cosmetics Control) test. The room was cleaned and tested regularly to ensure its cleanliness (Fig. 1).

The legal aspect is another fundamental requirement for cell-based therapy products. In China, the Guidelines for Human Somatic Cell Therapies and Quality Control of Cell-based Products stipulate that cell donation for clinical applications must comply with national requirements the same as for blood donation. In America, the Food and Drug Administration (FDA) "Tissue Donor Guidance (2007)" states that the potential tissue donor must test negative for a series of infectious microorganisms prior to donation. Screening for tissue donors who 


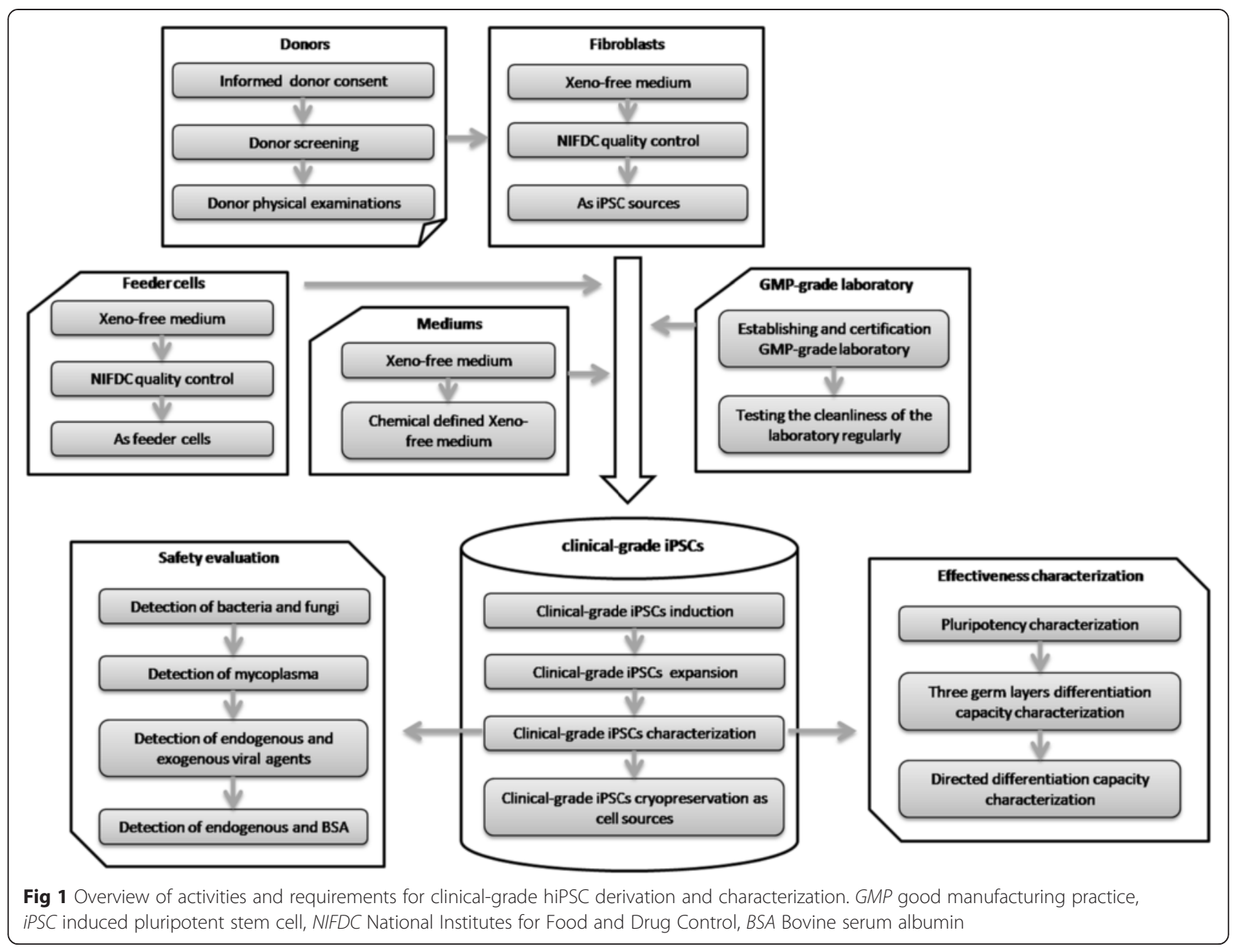

have signed the informed consent is therefore an important step to ensure the suitability of the cells generated from the donated tissue for clinical applications. After explaining the significance and potential applications of cells that would be generated from the donated tissue to children undergoing foreskin resection surgery and their parents, some patients and parents agreed to donate their tissues and signed the informed consent. The cases and medical reports of the patients were then strictly evaluated to assess their eligibility for donation. Finally, a 6-year-old boy's foreskin tissue was chosen (Fig. 1).

\section{Derivation of clinical-grade HFF cells}

Serum-containing medium are often used for fibroblast derivation and culture. To establish fibroblasts free of animal-source reagents in our laboratory, several types of Xeno-free or chemical defined human fibroblasts culture media were trialed (Data not shown). The result showed that FibroGRO ${ }^{\text {sm }}$ Xeno-Free Human Fibroblast Expansion Medium provided the optimum support for HFF cell growth. As a potential clinical-grade hiPSC and Xeno-free feeder cell source, biosecurity of the HFF has to be validated. In this case, a series of biosecurity-related projects were carried out. The results of these projects are presented in Additional file 2. The cell properties and safety are validated by the NIFDC in China.

\section{Derivation of clinical-grade hiPSCs}

The clinical-grade HFF cells of passage 8 were used for integration-free Sendai virus-based reprogramming. The reprogramming procedure was similar to the instructions of the CytoTune-iPS Sendai Reprogramming Kit except that Xeno-free media were used in our protocol. Pluriton ${ }^{\mathrm{Tu}}$ reprogramming medium is a type of Xeno-free medium which can efficiently support the generation of hiPSCs in mRNA-based reprogramming. The $\mathrm{X}$ medium, which was defined in our laboratory, could greatly improve the induction efficiency of pig and human iPSCs $[24,26]$ and could be configured with Xeno-free reagents. We proposed that clinical-grade hiPSCs could also be efficiently derived in pluriton $^{\text {Tw }}$ reprogramming medium and $\mathrm{X}$ medium, and thus both media were utilized in the reprogramming process. The hiPSC colonies emerged at day 12 after infection and were picked up 5 days later to generate 
stable cell lines (Fig. 2a). The reprogramming efficiency of $\mathrm{X}$ medium was almost three times that of the pluriton ${ }^{\text {th }}$ reprogramming medium (data not shown).

\section{Clinical-grade hiPSCs express specific pluripotency markers}

In total, 20 clinical-grade hiPSC lines were generated and two random lines were selected for further characterization. Both clinical-grade hiPSC lines exhibited morphology consistent with that of hESCs and could be subsequently passaged on clinical-grade HFF feeder layers (Fig. 2b; and Figure S1A in Additional file $3)$. These cells expressed high levels of alkaline phosphatase (Fig. 2c; and Figure S1B in Additional file 3) and maintained normal karyotypes when being characterized at passage 20 (Fig. 2d; and Figure S1C in Additional file 3). Real-time PCR results showed that the two cell lines expressed pluripotent genes Oct4 (Pou5f1), Sox2, Nanog, Rex1, and E-Cadherin at similar levels to hESCs (Fig. 2e). Immunofluorescence results showed that the two cell lines expressed pluripotency markers OCT4, SOX2, SSEA-3, SSEA-4, and TRA-1-60 and did not express differentiation marker SSEA-1 (Fig. 2f; and Figure S1D in Additional file 3).

Chemically defined E8 medium and vitronectin-coated surfaces have been validated for the support of hiPSC culture. Therefore, to avoid hiPSC cell culture instability stemming from variable human sourced albumin batches, E8 medium and vitronectin-coated surfaces were used in the culture of these two lines. Both lines grew well without major differentiation in E8 medium (Fig. 2b, h; and Figure S1A in Additional file 3). The percentages of SSEA-4, TRA-1-60, and TRA-1-81 expression were higher than 90 $\%$, whereas less than $1 \%$ of SSEA-1 was expressed (Fig. 2i).

PCR results showed that both lines were integrationfree (Fig. 2g) and all of the four Sendai virus vectors were gradually lost in the cytoplasm (Fig. $2 \mathrm{~h}$ ).

\section{Clinical-grade hiPSCs can differentiate into all three germ layers}

The EB and teratoma formation assays are commonly used to evaluate the differentiation ability of hPSCs. Both of the clinical-grade hiPSC lines were grown in suspension in clinical-grade hESC basic medium. Eight days later, they all formed round EBs (Fig. 3a; and Figure S1E in Additional file 3). RT-PCR analysis showed that the EBs expressed markers of all three germ layers, including ectoderm (Gad1 and Pax6), mesoderm (Enolase and Ostenectin), and endoderm (Nicastrin and Alphafetoprotein) (Fig. 3b). The teratoma formation assay was performed by injection of the two hiPSC lines to the testis of SCID mice separately. Two months later, teratomas were visualized in all injected testes. Histological analysis revealed that the teratomas comprised tissues of all three germ layers (Fig. 3c; and Figure S1F in Additional file 3).

\section{Clinical-grade hiPSCs can directly differentiate to functional neural cells and cardiomyocytes in Xeno-free media}

hPSCs including hESCs and hiPSCs cannot be used directly in cell replacement therapies as they may induce tumor formation. However, terminally differentiated functional cells derived from hiPSCs can be used for clinical applications. Therefore it is important to evaluate the differentiation ability of our clinical-grade hiPSCs into specific cell types under Xeno-free conditions. Neural cell and cardiomyocyte differentiations were chosen for this purpose.

For neural cell differentiation, hiPSCs were directed to forebrain dopamine neurons following a series of spatial and temporal specific signal inductions according to the neural lineage development process [25]. Twenty-three days after differentiation, the neurons expressed neural marker TUJ1 and dopamine neural marker TH1 (Fig. 3d).

For cardiomyocyte differentiation, the Car-CDM medium modified in our laboratory was used. The differentiation process followed a temporal WNT signal activation and inhibition as described previously [27]. Beating cardiomyocytes were visible 12 days after differentiation and cardiomyocyte specific markers NKX2.5 and CTNT were detected by immunofluorescence staining (Fig. 3e).

\section{Clinical-grade hiPSCs are biologically safe}

To evaluate whether our clinical-grade hiPSCs are biologically safe, we performed a series of tests according to the Guidelines for Human Somatic Cell Therapies and Quality Control of Cell-based Products. The results showed that the cells were negative for mycoplasma and free of serious pathogenic microorganisms such as HIV and HPV (Additional file 2). The endotoxin level of the hiPSC culture medium met the requirements defined in the Pharmacopoeia of the People's Republic of China, 2010 edition, Volume III (Additional file 2). To detect other types of unknown pathogenic microorganisms, we injected the hiPSCs into the chorioallantoic and yolk sac of chicks. After 5 and 10 days of incubation separately, the chicks were still alive (Additional file 2), which indicated that the hiPSCs were not infected with pathogenic microorganisms.

\section{Discussion}

Clinical-grade hiPSCs and GMP environments are essential in order to ensure the safety, effectiveness, traceability, and reproducibility of hiPSC-based clinical trials or therapies $[16,28]$. To avoid the risk of cross- 


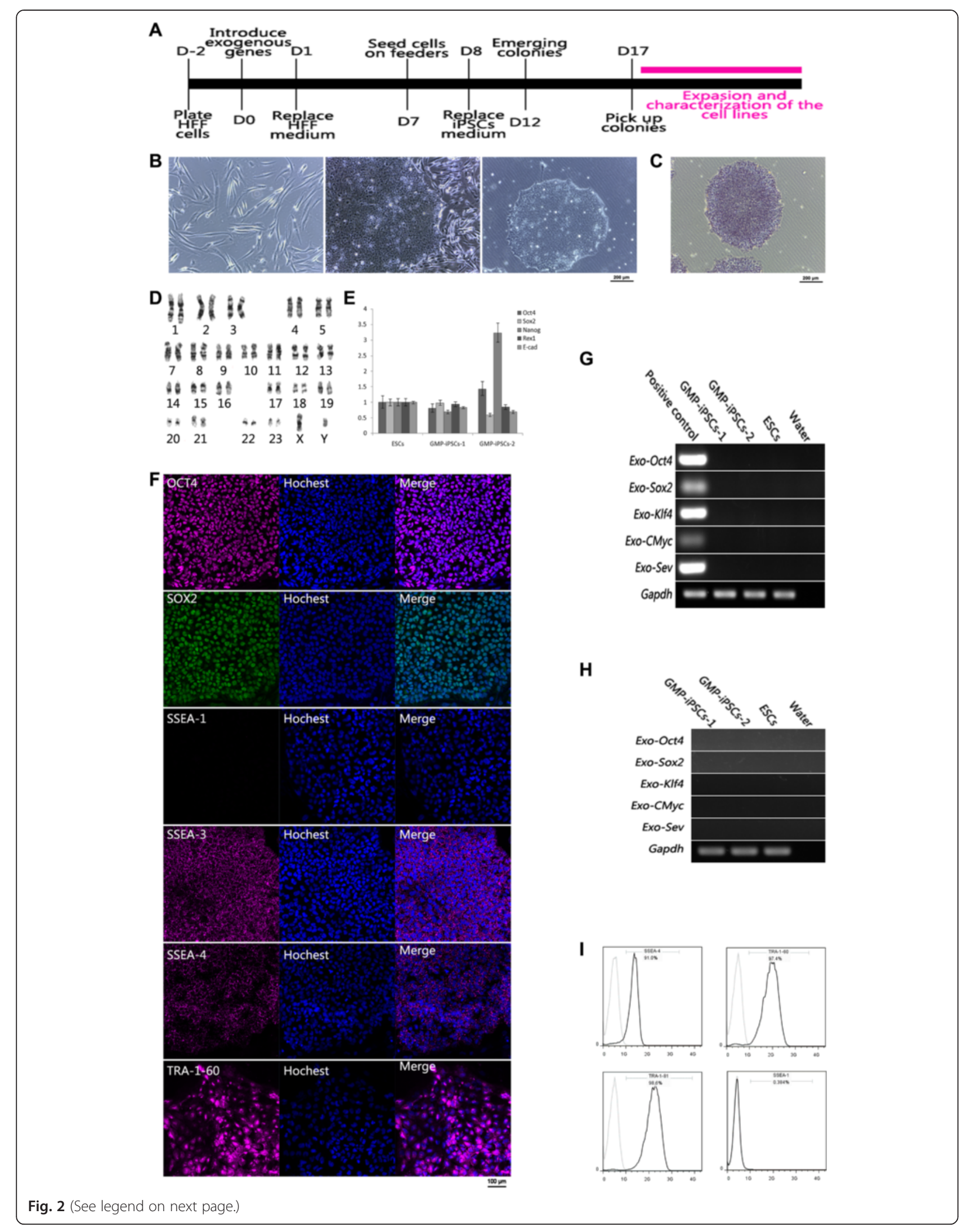


(See figure on previous page.)

Fig. 2 Reprogramming of clinical-grade HFF cells into clinical-grade hiPSCs and characterization of their pluripotency. a Time course of clinical-grade hiPSC generation. $\mathbf{b}$ Morphology of clinical-grade HFF cells, and clinical-grade hiPSCs grown on HFF feeder cells and in feeder-free conditions. Scale bar, $200 \mu \mathrm{m}$. c Clinical-grade hiPSCs express alkaline phosphatase. Scale bar, $200 \mu \mathrm{m}$. d Karyotyping results of one of the clinical-grade hiPSC lines. e mRNA expression levels of pluripotency-related genes of the clinical-grade hiPSCs relative to hESCs. $\mathbf{f}$ Immunofluorescence results of one of the clinical-grade hiPSC lines. The hiPSCs express pluripotency markers OCT4, SOX2, SSEA-3, SSEA-4, and TRA-1-60 at the protein level and do not express differentiation marker SSEA-1. Scale bar, $100 \mu \mathrm{m} . \mathbf{g}$ PCR analysis results show that the clinical-grade hiPSCs did not express Sendai virus exogenous genes. $\mathbf{h}$ PCR analysis results show that the Sendai virus genes did not integrate into the genomic of the clinical-grade hiPSCs. i Flow cytometry analysis of SSEA-1, SSEA-4, TRA-1-60, and TRA-1-81 expression of one of the clinical-grade hiPSC lines growing without feeders. D day, ESC embryonic stem cell, GMP good manufacturing practice, HFF human foreskin fibroblast, iPSC induced pluripotent stem cell, SSEA stage-specific embryonic antigen

species infectious agent transfer, the usage of animalsource reagents in cell handling process is not recommended [29].

The hESCs used in the clinical trials approved by the US FDA as mentioned earlier were not derived under GMP conditions. Instead they were converted from research-grade lines which were derived and maintained in the presence of animal products [28]. It is unclear whether this protocol can be applied in hiPSCs. After all, it is far easier to obtain cell sources for hiPSCs than for hESCs. Recently, several groups reported that they have successfully derived clinical-grade or Xeno-free hiPSCs. However, the derivation of hiPSCs was not entirely Xeno-free [21-23] or under GMP conditions [20]. To our knowledge, our study is the first to report successful derivation of clinical-grade hiPSC lines completely under GMP conditions in Xeno-free reagents. These hiPSC lines may be a valuable source for future clinical trials or therapies.

Oncogenicity is a major concern for the application of hiPSCs in clinical settings. Nevertheless, an increasing number of studies in mouse models suggest that iPSCs are safe for transplantation [30]. For example, Tsuji et al. [30] have reported no tumor formation after the transplantation of iPSC-derived neural spheres into SCID mouse brain. The process of obtaining iPSCs is complex, however, involving the reconstruction of cellular epigenetic states. Assessment of genomic integrity and epigenetic variations of hiPSCs is therefore very important to ensure their safety. To date, the induction of genomic and epigenetic variations in iPSCs is still questionable. Hussein et al. [31] reported that there are more chromosomal copy number variations in iPSCs compared with embryonic stem cells and their parental cells, while Abyzov et al. [32] proved that reprogramming did not lead to copy number variations using single-cell sequencing. Interestingly, a few groups reported abnormal genomic imprinting and methylation in iPSCs [33, 34]. The quality of each iPSC line varies and thus it is important to define a series of criteria from the genomic and epigenetic level to evaluate the safety of the iPSCs before their clinical application. This requires joined efforts from scientists all over the world. The results of clinical trials in Japan will give us some clues.

Reprogramming efficiency is another important factor for future hiPSC-based therapies because parental cells from old patients which are difficult to be reprogrammed may be required. We compared the reprogramming efficiency of two Xeno-free mediums and found that the number of colonies yielded from $\mathrm{X}$ medium, defined in our laboratory, are three times that from pluriton ${ }^{\mathrm{Tm}}$ reprogramming medium, suggesting that our $\mathrm{X}$ medium could be used as a high-efficiency clinical-grade reprogramming medium.

Envisaging the potential translation from hiPSCs to cell replacement therapies, Xeno-free and GMP conditions are essential for the differentiation of hiPSCs into functional cells. We demonstrated the feasibility of differentiating one clinical-grade hiPSC line to functional neurons and cardiomyocytes in Xeno-free mediums according to the monolayer differentiation methods. As for monolayer differentiation, each hPSC line has an optimal differentiation method, and hence further work will be focused on optimizing the monolayer differentiation method to obtain functional neurons, cardiomyocytes, and other cell types from the clinical-grade hiPSC lines.

The incidence of degenerative diseases such as Parkinson's disease and Alzheimer's disease which cannot be treated effectively by traditional medical methods is increasing worldwide. Theoretically, replacement of degenerated cells with normal functional cells in patients paves the way for new treatment strategies. hiPSCs can provide an unlimited number of cells for autologous or closely HLA-matched transplantation upon differentiation which significantly reduces the risk of host immune response in patients. Only by standardizing and normalizing all processes during stem cell-based therapies can we ensure therapy safety and obtain objective and reliable results. These processes involve acquisition, cultivation, characterization, and purification of stem cells, testing tumorigenicity and immunogenicity of stem cells, in vivo reinfusion, efficacy evaluation of stem cells, and so on. In the near future, stem cell-based therapies will create a landmark achievement in the field of regenerative 


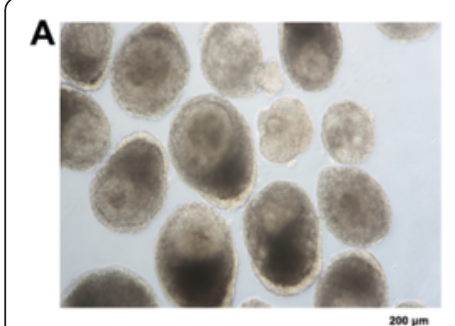

B

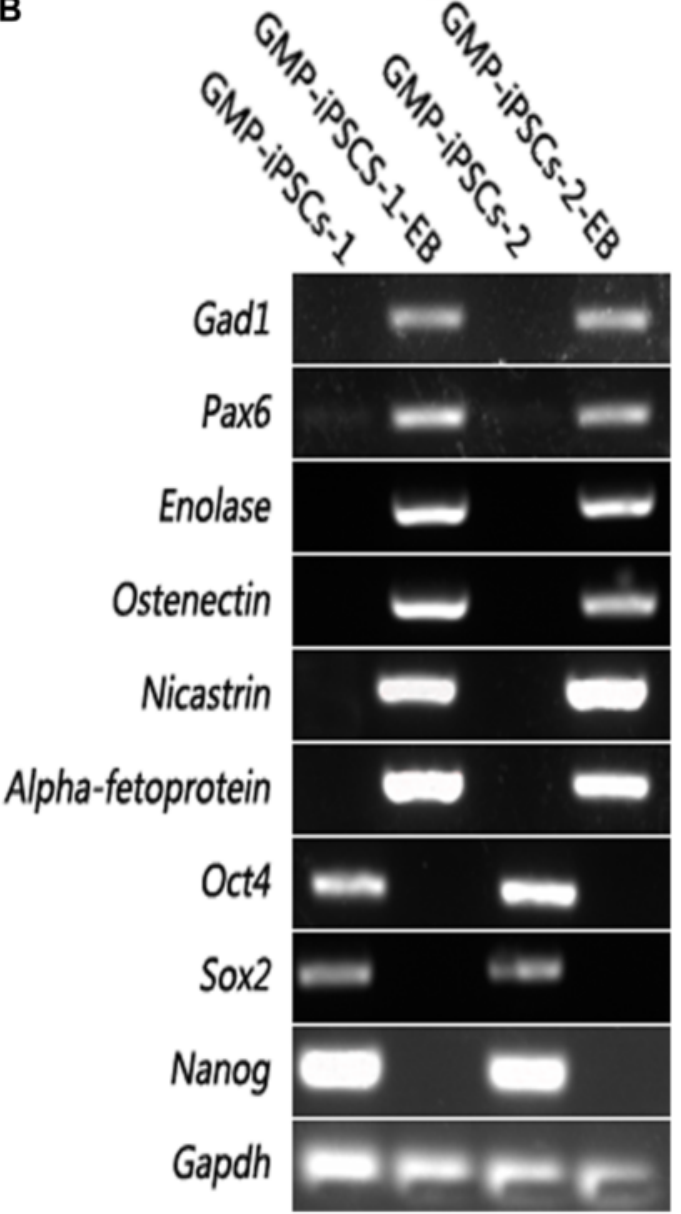

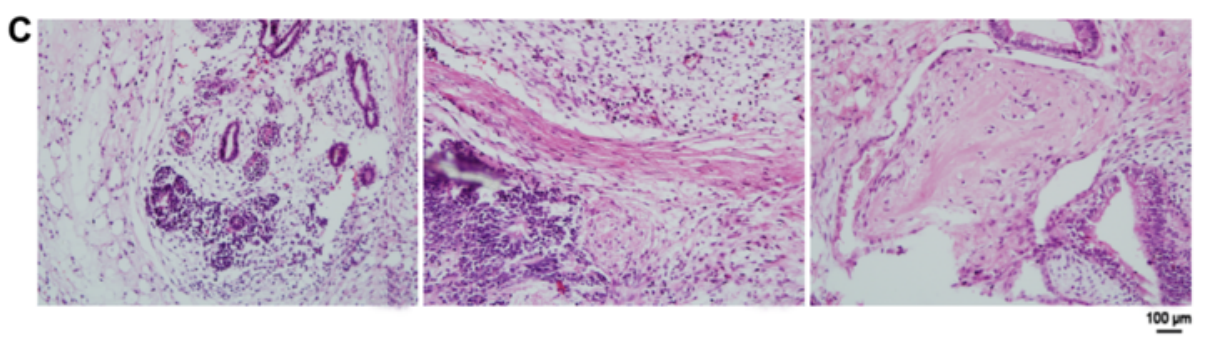

$100 \mathrm{vm}$
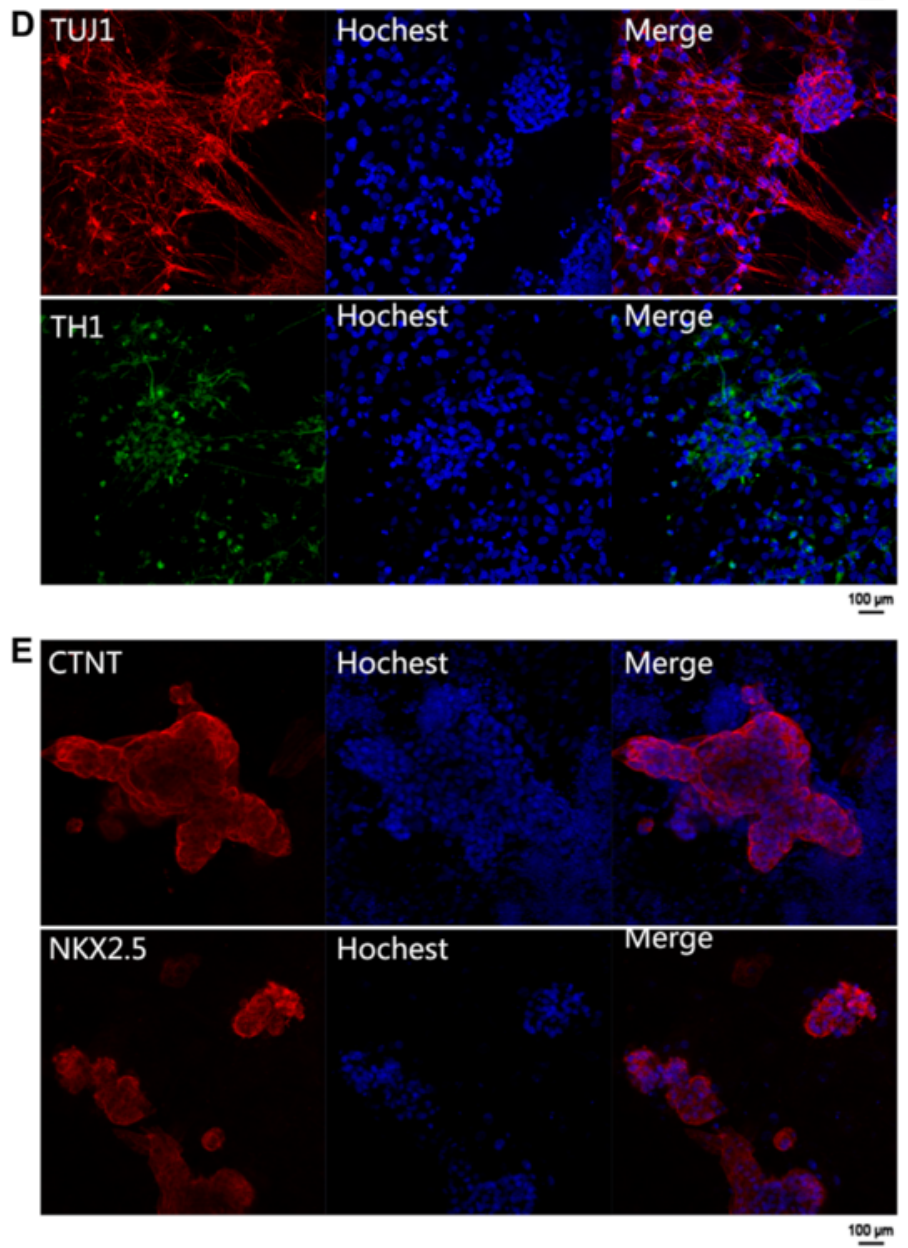

Fig 3 Clinical-grade hiPSCs can randomly differentiate into all three germ layers and directly differentiate into functional neural cells and cardiomyocytes in Xeno-free media. a Morphology of EBs at day 8 derived from one clinical-grade hiPSC line. Scale bar, $200 \mu \mathrm{m}$. b Gene expression profile for germ-layer marker genes of the day 8 EBs, ectoderm (Gad1 and Pax6), mesoderm (Enolase and Osteonectin), endoderm (Nicastrin and Alpha-fetoprotein), and pluripotency (Oct4, Sox2 and Nanog). c Hematoxylin and eosin staining of teratoma derived from one clinical-grade hiPSC line. Scale bar, 100 um. The teratomas contain tissues of all three germ layers. $\mathbf{d}$ Immunofluorescence results of neural cells differentiated from one clinical-grade hiPSC line. The neural cells express TH1 and TUJ1 at protein level. Scale bar, $100 \mu m$. e Immunofluorescence results of cardiomyocytes differentiated from one clinical-grade hiPSC line. The cardiomyocytes express NKX2.5 and CTNT at protein level. Scale bar, $100 \mu \mathrm{m}$. EB embryoid body, GMP good manufacturing practice, iPSC induced pluripotent stem cell

medicine and provide the theoretical basis and technical supports for conquering particular "human ills" by following strict regulations.

\section{Conclusions}

hiPSCs hold great promise in regenerative medicine. To ensure clinical safety and obtain reliable therapeutic results, standardized and normalized processes must be followed during hiPSC-based therapies. Herein, integrationfree clinical-grade hiPSC lines were derived under GMPcontrolled conditions and with Xeno-free reagent culture in line with the current guidance of international and national evaluation criteria, and proved to be biologically safe and capable of differentiating into functional neural 
cells and cardiomyocytes in Xeno-free culture media. These results provide that the clinical-grade hiPSC lines could be valuable sources for future hiPSC-based clinical trials or therapies, especially in neural and heart-related diseases.

\section{Additional files}

Additional file 1: Table S1. Presenting primer sequences for RT-PCR. (DOC $37 \mathrm{~kb}$ )

Additional file 2: Table S2. Presenting sterility and pathogen testing in the two clinical-grade hiPSC lines. (DOC $33 \mathrm{~kb}$ )

Additional file 3: Figure S1. Showing characterization of another clinical-grade hiPSC line. A Morphology of one clinical-grade hiPSC grown on human foreskin fibroblasts feeder cells and in feeder-free conditions. Scale bar, $200 \mu \mathrm{m}$. B Clinical-grade hiPSCs express alkaline phosphatase. Scale bar, $200 \mu \mathrm{m}$. C Karyotyping results of one clinical-grade hiPSC line. $D$ Immunofluorescence results of one clinical-grade hiPSC line. The hiPSCs express pluripotency markers OCT4, SOX2, SSEA-3, SSEA-4, and TRA-1-60 at the protein level and do not express differentiation marker SSEA-1. SSEA stage-specific embryonic antigen. Scale bar, $100 \mu \mathrm{m}$. E Morphology of EBs at day 8 derived from one clinical-grade hiPSC line. Scale bar, $200 \mu \mathrm{m}$. F Hematoxylin and eosin staining of teratoma derived from one clinical-grade hiPSC line. Scale bar, $100 \mu \mathrm{m}$. The teratomas contain tissues of all three germ layers. (TIFF $9074 \mathrm{~kb}$ )

\section{Abbreviations}

BDNF: Brain-derived neurotrophic factor; bFGF: Basic fibroblast growth factor; DMEM: Dulbecco's modified Eagle's medium; EB: Embryoid body; EBV: Epstein-Barr virus; EDTA: Ethylenediamine tetraacetic acid; FDA: Food and Drug Administration; FITC: Fluorescein isothiocyanate; GDNF: Glial cell line-derived neurotrophic factor; GMP: Good manufacturing practice; HAV: Hepatitis A virus; HBV: Hepatitis B virus; HCMV: Human cytomegalovirus; HCV: Hepatitis C virus; hESC: Human embryonic stem cell; HFF: Human foreskin fibroblast; hiPSC: Human induced pluripotent stem cell; HIV: Human immunodeficiency virus; hLif: Leukemia inhibitory factor; hPSC: Human pluripotent stem cell; HPV: Human papilloma virus; HTLV-I: Human Tlymphotropic virus I; IACUC: Institutional Animal Care and Use Committee; JCV: John Cunningham virus; KOSR: Knockout serum replacement; NEAA: None essential amino acid; NIFDC: National Institutes for Food and Drug Control; NIH: National Institutes of Health; RPE: Retinal pigmented epithelial; SCID: Severe combined immunodeficiency; TGF $\beta 3$ : Transforming growth factor type $\beta 3$.

\section{Competing interests}

The authors declare that they have no competing interests.

\section{Authors' contributions}

JWa designed the research, drafted the manuscript, and derived and characterized the clinical-grade hiPSCS. JH designed the research and revised the whole manuscript. DB, XL, and QG participated in the derivation and culture of clinical-grade hiPSCs and HFF cells, and revised the Methods section of the manuscript. WH, LeW, YT, PH, and ZheL participated in the differentiation and characterization of neural cells and cardiomyocytes, and revised the Results section of the manuscript. KX evaluated the biological safety of the clinical-grade HFF cells and hiPSCs, and revised the corresponding part of the manuscript. YJ, $J W u, W L, L L$, and LiW revised and drafted the discussion part of the manuscript. ZhoL and QZ designed the research, revised and edited the manuscript, and gave final approval of the version. All authors read and approved the final manuscript.

\section{Acknowledgements}

This study was supported by grants from China National Basic Research Program of China (Grant No. 2013CB967102), the "Strategic Priority Research Program" of the Chinese Academy of Sciences (Grant No. XDA01030506), and the International S\&T Cooperation Program of China (Grant No. XDA01030506 2013DFG30680).

\section{Author details}

${ }^{1}$ State Key of Stem Cells and Laboratory of Reproductive Biology, Institute of Zoology, Chinese Academy of Sciences, Beijing 100101, China. ${ }^{2}$ College of Life Science, Northeast Agricultural University of China, Harbin 150030, China.

${ }^{3}$ Graduate School of Chinese Academy of Sciences, Beijing 100049, China.

Received: 26 March 2015 Revised: 31 March 2015

Accepted: 16 October 2015 Published online: 12 November 2015

\section{References}

1. Hayden EC. Funding windfall rescues abandoned stem-cell trial. Nature. 2014;510:18

2. Good practice. Nature. 2014;510:187-8. http://www.nature.com/news/goodpractice-1.15383.

3. Schwartz SD, Hubschman JP, Heilwell G, Franco-Cardenas V, Pan CK, Ostrick RM, et al. Embryonic stem cell trials for macular degeneration: a preliminary report. Lancet. 2012;379:713-20.

4. Song $P$, Inagaki $Y$, Sugawara $Y$, Kokudo N. Perspectives on human clinical trials of therapies using iPS cells in Japan: reaching the forefront of stem-cell therapies. Biosci Trends. 2013;7:157-8.

5. Takahashi K, Tanabe K, Ohnuki M, Narita M, Ichisaka T, Tomoda K, et al. Induction of pluripotent stem cells from adult human fibroblasts by defined factors. Cell. 2007;131:861-72

6. Yu J, Vodyanik MA, Smuga-Otto K, Antosiewicz-Bourget J, Frane JL, Tian S, et al. Induced pluripotent stem cell lines derived from human somatic cells. Science. 2007;318:1917-20.

7. Tong M, Lv Z, Liu L, Zhu H, Zheng QY, Zhao XY, et al. Mice generated from tetraploid complementation competent iPS cells show similar developmental features as those from ES cells but are prone to tumorigenesis. Cell Res. 2011;21(11):1634-7.

8. Merling RK, Sweeney CL, Choi U, De Ravin SS, Myers TG, Otaizo-Carrasquero $F$, et al. Transgene-free iPSCs generated from small volume peripheral blood nonmobilized CD34+ cells. Blood. 2013;121:e98-107.

9. Mack AA, Kroboth S, Rajesh D, Wang WB. Generation of induced pluripotent stem cells from CD34+ cells across blood drawn from multiple donors with non-integrating episomal vectors. PLoS One. 2011;6:e27956.

10. Warren L, Manos PD, Ahfeldt T, Loh YH, Li H, Lau F, et al. Highly efficient reprogramming to pluripotency and directed differentiation of human cells with synthetic modified mRNA. Cell Stem Cell. 2010;7:618-30.

11. Jia F, Wilson KD, Sun N, Gupta DM, Huang M, Li Z, et al. A nonviral minicircle vector for deriving human iPS cells. Nat Methods. 2010;7:197-9.

12. Miyoshi $N$, Ishii $H$, Nagano $H$, Haraguchi N, Dewi DL, Kano $Y$, et al. Reprogramming of mouse and human cells to pluripotency using mature microRNAs. Cell Stem Cell. 2011;8:633-8.

13. Kim D, Kim CH, Moon Jl, Chung YG, Chang MY, Han BS, et al. Generation of human induced pluripotent stem cells by direct delivery of reprogramming proteins. Cell Stem Cell. 2009;4:472-6.

14. Jonlin EC. Differing standards for the NIH Stem Cell Registry and FDA approval render most federally funded hESC lines unsuitable for clinical use. Cell Stem Cell. 2014;14:139-40.

15. Unger C, Skottman H, Blomberg P, Dilber MS, Hovatta O. Good manufacturing practice and clinical-grade human embryonic stem cell lines. Hum Mol Genet. 2008;17:R48-53.

16. Andrews PW, Cavanagro J, Deans R, Feigel E, Horowitz E, Keating A, et al. Harmonizing standards for producing clinical-grade therapies from pluripotent stem cells. Nat Biotechnol. 2014;32:724-6.

17. Martin MJ, Muotri A, Gage F, Varki A. Human embryonic stem cells express an immunogenic nonhuman sialic acid. Nat Med. 2005;11:228-32.

18. European Medicines Agency. CAT Secretariat \& US Food and Drug Administration. Regen Med. 2011;6:90-6. http://www.futuremedicine.com/ doi/pdf/10.2217/rme.11.86.

19. Devito L, Petrova A, Miere C, Codognotto S, Blakely N, Lovatt A, et al. Cost-effective master cell bank validation of multiple clinical-grade human pluripotent stem cell lines from a single donor. Stem Cells Transl Med. 2014;3:1116-24.

20. Chen G, Gulbranson DR, Hou Z, Bolin JM, Ruotti V, Probasco MD, et al. Chemically defined conditions for human iPSC derivation and culture. Nat Methods. 2011:8:424-9.

21. Awe JP, Lee PC, Ramathal C, Vega-Crespo A, Durruthy-Durruthy J, Cooper A, et al. Generation and characterization of transgene-free human induced 
pluripotent stem cells and conversion to putative clinical-grade status. Stem Cell Res Ther. 2013;4:87.

22. Rajala K, Lindroos B, Hussein SM, Lappalainen RS, Pekkanen-Mattila M, Inzunza J, et al. A defined and xeno-free culture method enabling the establishment of clinical-grade human embryonic, induced pluripotent and adipose stem cells. PLoS One. 2010;5:e10246.

23. Durruthy-Durruthy J, Briggs SF, Awe J, Ramathal CY, Karumbayaram S, Lee PC, et al. Rapid and efficient conversion of integration-free human induced pluripotent stem cells to GMP-grade culture conditions. PLoS One. 2014;9:e94231.

24. Wang J, Gu Q, Hao J, Bai D, Liu L, Zhao X, et al. Generation of induced pluripotent stem cells with high efficiency from human umbilical cord blood mononuclear cells. Genomics Proteomics Bioinformatics. 2013;11:304-11.

25. Kriks S, Shim JW, Piao J, Ganat YM, Wakeman DR, Xie Z, et al. Dopamine neurons derived from human ES cells efficiently engraft in animal models of Parkinson's disease. Nature. 2011;480:547-51.

26. Gu Q, Hao J, Hai T, Wang J, Jia Y, Kong Q, et al. Efficient generation of mouse ESCs-like pig induced pluripotent stem cells. Protein Cell. 2014;:5:338-42.

27. Lian X, Hsiao C, Wilson G, Zhu K, Hazeltine LB, Azarin SM, et al. Robust cardiomyocyte differentiation from human pluripotent stem cells via temporal modulation of canonical Wnt signaling. Proc Natl Acad Sci U S A. 2012;109:E1848-57.

28. Ilic D, Stephenson E. Promises and challenges of the first clinical-grade induced pluripotent stem cell bank. Regen Med. 2013;8:101-2.

29. Abbasalizadeh S, Baharvand H. Technological progress and challenges towards CGMP manufacturing of human pluripotent stem cells based therapeutic products for allogeneic and autologous cell therapies. Biotechnol Adv. 2013;31:1600-23.

30. Tsuji O, Miura K, Fujiyoshi K, Momoshima S, Nakamura M, Okano H. Cell therapy for spinal cord injury by neural stem/progenitor cells derived from iPS/ES cells. Neurotherapeutics. 2011;8:668-76.

31. Hussein SM, Batada NN, Vuoristo S, Ching RW, Autio R, Narva E, et al. Copy number variation and selection during reprogramming to pluripotency. Nature. 2011;471:58-62.

32. Abyzov A, Mariani J, Palejev D, Zhang Y, Haney MS, Tomasini L, et al. Somatic copy number mosaicism in human skin revealed by induced pluripotent stem cells. Nature. 2012;492:438-42.

33. Hiura H, Toyoda M, Okae H, Sakurai M, Miyauchi N, Sato A, et al. Stability of genomic imprinting in human induced pluripotent stem cells. BMC Genet. 2013;14:32.

34. Lister R, Pelizzola M, Kida YS, Hawkins RD, Nery JR, Hon G, et al. Hotspots of aberrant epigenomic reprogramming in human induced pluripotent stem cells. Nature. 2011;471:68-73.

\section{Submit your next manuscript to BioMed Central and take full advantage of:}

- Convenient online submission

- Thorough peer review

- No space constraints or color figure charges

- Immediate publication on acceptance

- Inclusion in PubMed, CAS, Scopus and Google Scholar

- Research which is freely available for redistribution 